\title{
Elevated pre-surgical CAI5-3: does it predict the short-term disease-free survival of breast cancer patients without distant metastasis?
}

This article was published in the following Dove Press journal: International Journal of Women's Health

\author{
Lakmini KB Mudduwa' \\ Gaya B Wijayaratne ${ }^{2}$ \\ Harshini H Peiris ${ }^{3}$ \\ Shania N Gunasekera' \\ Deepthika \\ Abeysiriwardhana' \\ Nimsha Liyanage'
}

'Department of Pathology, Faculty of Medicine, University of Ruhuna, Galle, Sri Lanka; ${ }^{2}$ Department of Microbiology, Faculty of Medicine, University of Ruhuna, Galle, Sri Lanka; ${ }^{3}$ Medical Laboratory Science Degree Program, Faculty of Medicine, University of Ruhuna, Galle, Sri Lanka

Correspondence: Lakmini KB Mudduwa Department of Pathology, Faculty of Medicine, University of Ruhuna, PO Box 70, Galle 80000 , Sri Lanka $\mathrm{Tel}+947 \mid 426$ I883

Fax +94 912222314

Email lakminimudduwa@yahoo.com
Background: CA15-3 is the most commonly used tumor marker in breast cancer. Its prognostic role has been described in the metastatic setting, but the role of pre-surgical CA15-3 in the assessment of patients with breast cancer without metastasis has not been substantiated yet.

Methodology: From February 2014 for a 2-year period, this prospective study included all patients who were diagnosed with primary breast cancer and underwent surgery at a tertiary care hospital. The serum level of CA15-3 was assessed on a pre-surgical blood sample and later at the 3-, 6-, 9-, and 12-month follow-up by enzyme-linked immunosorbent assay. Disease-free survival (DFS) was analyzed with a Kaplan-Meier model and log-rank test.

Results: We enrolled 195 patients (mean age \pm SD 57.84 years \pm 13.819 , range, 28-95) with breast cancer. The prevalence of elevated ( $\geq 30 \mathrm{U} / \mathrm{mL}$ ) pre-surgical CA15-3 was $35.9 \%$, and it reduced to $14.3 \%$ at 3 months after mastectomy. Pre-surgical CA15-3 had a significant association only with the size of the tumor $(p=0.047)$. Patients who did not have elevated pre-surgical CA15-3 ( $\geq 30 \mathrm{U} / \mathrm{mL}$ ) had the best short-term DFS, and it was worst when the pre-surgical CA15-3 was $>100 \mathrm{U} / \mathrm{mL}(p=0.041)$.

Conclusion: Elevated pre-surgical CA15-3 is a predictor of poor short-term DFS of patients with breast cancer without distant metastasis.

Keywords: breast cancer, pre-surgical CA15-3, short-term disease-free survival, prognosis

\section{Background}

CA15-3 was first recognized as a breast-associated antigen although it is currently described as a mucin and is also called MUC-1. ${ }^{1}$ It was first isolated in human milk. ${ }^{2}$ The human mucin (MUC) family comprises 21 members, and MUC1 is one of them. ${ }^{3}$ The CA15-3 assay detects soluble products of the $M U C-1$ gene. MUC-1 is a transmembrane molecule expressed in most glandular epithelial cells. ${ }^{4}$ MUC-1 is overexpressed in breast cancer compared to normal breast epithelial cells. ${ }^{1}$ MUC-1 is upregulated and aberrantly glycosylated in many other carcinomas as well. ${ }^{2}$ Aberrant MUC-1 glycosylation is found to play a role in the immunosurveillance of cancer and, therefore, is a potential target for cancer therapy. ${ }^{4}$

CA15-3 - the soluble product of the MUC1 gene - is the most widely used serum marker of breast cancer. ${ }^{5,6}$ However, it is not recommended for screening because of low sensitivity. ${ }^{7}$ Elevated CA15-3 level is predictive of poor response to chemotherapy in locally advanced disease and is found in most patients with metastatic breast cancer. ${ }^{5,6,8}$ The American Society of Clinical Oncology recommends CA15-3 for monitoring patients with metastatic disease during active therapy. It recommends CA27.29 or 
CA15-3 to be used in conjunction with diagnostic imaging, history, and physical examination, but not alone. ${ }^{7,9}$

The value of pretreatment CA15-3 in predicting prognosis has been recorded by several studies, most of which have been done in Europe. Most of them found that elevated presurgical CA15-3 is associated with poor disease-free survival (DFS). ${ }^{6}$ The value of CA15-3 in advanced breast cancer is addressed in most publications, and it is often claimed that its value in the non-metastatic setting is limited. Pre-surgical CA15-3 assessment has not been included yet in the breast cancer management guidelines because current evidence favoring it as a prognostic marker is insufficient.

Prognostic markers that are routinely used in breast cancer are tissue based and need a sample of tissue for assessment. Therefore, a serum marker assessment which is noninvasive, reproducible, and an objective too $1{ }^{10}$ is valuable in prognostication. Therefore, this study was conducted to evaluate the value of the pre-surgical assessment of CA15-3 in predicting the short-term DFS of breast cancer patients without distant metastasis. Moreover, we intended to assess whether the elevated CA15-3 levels decline after surgery.

\section{Materials and methods}

This prospective study included all patients diagnosed with breast cancer at the teaching hospital of the Southern province from February 2014 for a 2-year period who gave written informed consent for study participation. Ethical approval was obtained from the Ethical Review Committee of the Faculty of Medicine, University of Ruhuna, Sri Lanka, before commencing the study. All study participants were followed up at the clinic until August 2016. Patients who were given neoadjuvant chemotherapy and those with metastasis at presentation were excluded. A $3 \mathrm{~mL}$ venous blood sample was collected prior to mastectomy from each patient. Venous blood samples $(3 \mathrm{~mL})$ were collected at 3-month intervals for 1 year post surgery. Serum was separated and stored in a refrigerator at $-70^{\circ} \mathrm{C}$ until the samples were thawed and processed batch-wise for the CA15-3 assay by enzyme-linked immunosorbent assay (ELISA).

AccuDiag ${ }^{\text {TM }}$ CA15-3 ELISA Kit from Diagnostic Automation/Cortez Diagnostics Inc., Calabasas, CA, USA, was used for this study. This ELISA test is a two-site solidphase enzyme immunoassay. The molecules of CA15-3 are "sandwiched" between two monoclonal antibodies - one coated to the bottom of the wells of the microtiter plates and the other linked to the horseradish peroxidase (enzyme conjugate). After incubation and washing, the enzymatic reaction developed a color in proportion to the amount of CA15-3 molecules present in the assay.
Each batch of test included a known positive and a negative control and six standard sera with CA15-3 values of 0,15 , $30,60,120$, and $240 \mathrm{U} / \mathrm{mL}$ that were used to plot the graph to interpret CA15-3 values of the test samples. The test was done in duplicate for each serum sample, and the mean was taken as the CA15-3 value. The minimum detectable CA15-3 level of this assay was $5.0 \mathrm{U} / \mathrm{mL}$. A value of $\geq 30 \mathrm{U} / \mathrm{mL}$ was considered to be indicative of elevated CA15-3.5,11 All samples that gave a CA15-3 value between 25 and $35 \mathrm{U} / \mathrm{mL}$ were repeated and confirmed.

\section{Tissue microarray construction and immunohistochemical assessment}

Tumor blocks of all patients enrolled in the study that were used for routine estrogen and progesterone receptor (ER and $\mathrm{PgR}$ ) and HER2 assessment were collected for tissue microarray (TMA) construction. The best representative tumor region with minimum fixation artifacts which included the invasive tumor front was circled on the hematoxylin and eosin (H\&E)-stained slides of each case. The H\&E slide was superimposed on the donor block to identify the area in the tissue block to be punched for 2-mm diameter tissue cores using TMA builder ${ }^{\mathrm{TM}}$ (Thermo Fisher Scientific). The cores were transposed into the recipient TMA wax mold that was prepared previously and contained 24 pits.

ER $\alpha$ clone 1D5 (Dako-M7047), PgR (Dako-M3569), and HER2 (Dako-A0485) were used for immunohistochemical (IHC) staining for the corresponding markers on whole sections as is done routinely. Antibodies against Claudin3 (Abcam-ab15102), Ki67 (Dako M7240), CK 5/6 (Dako M7237), and EGFR (Dako M3563) were used with the secondary antibody (Dako Real EnVision ${ }^{\mathrm{TM}}$ ) for IHC staining of all breast cancers in TMAs.

An Allred score $\leq 2$ for ER and PgR, together with a score of 0 or +1 or +2 for HER2, was considered the criterion for categorizing a case as triple-negative breast cancer (TNBC). ER and PgR were considered positive when the Allred score for each was $>2$. Furthermore, HER2 equivocal staining (2+) was considered HER2 negative when fluorescence in situ hybridization (FISH) results confirming otherwise were not available. Each breast cancer was classified according to the molecular classification, depending on the expression of the mentioned IHC markers, based on the criterion specified in the St Gallen Guidelines 2013. ${ }^{12}$ A TNBC positive for either CK5/6 or EGFR was considered basal like. ${ }^{13,14}$

\section{Follow-up and outcomes}

Patients included in the study were prospectively followed up at the Oncology clinic for a maximum of 30 months. 
The mean follow-up time was $15.08 \pm 17.23$ months. The DFS was calculated from the date of surgery to the date of death or the date of diagnosis of the recurrence (local/distant metastasis) or the date of diagnosis of the contralateral breast cancer. ${ }^{15}$ Radiological and histopathological evidence was used to confirm the recurrence; the date of the said investigation was considered the date of recurrence. Patients who did not experience the relevant endpoint were censored at the last follow-up. ${ }^{15}$

\section{Statistical analysis}

The Pearson chi-square test was used to determine the association between serum CA15-3 and the clinicopathological features. The Kaplan-Meier model was used to estimate the DFS. The log-rank test was used to compare the survival of different groups. The Kaplan-Meier model for univariate, and the Cox regression model with backward stepwise factor retention method for multivariate, analyses were used to estimate the predictors of short-term DFS. A $p$-value $<0.05$ was considered significant in all analyses.

\section{Results}

\section{Clinicopathological profile of the study cohort}

A total of 195 patients with breast cancer were enrolled in this study. All subjects were female, with a mean age of 57.84 years ( $\mathrm{SD} \pm 13.819$, range, 28-95). Most patients were middle-aged or $>60$ years $(\geq 35$ years, $2.65 \%$; 36-60 years, $54.4 \%$; and $>60$ years, $43.1 \%$ ). The Nottingham Grade 2 was the most common grading in this study population (Grade 1, 14.6\%; Grade 2, 48.1\%; Grade 3, 37.3\%). The majority of the cohort was in TNM Stage II at presentation (stages I, 12.1\%; II, 54.4\%; III, 33.5\%). Lymph node metastasis was present at the time of presentation in $52.6 \%$ of patients. The majority had ER- (68.8\%) and PgR (59.1\%)-positive tumors. HER2 was overexpressed in $27.2 \%$ of patients (36 patients had HER2 equivocal expression and FISH data were unavailable). The Claudin-low breast cancer comprised $11 \%$ of the tested samples. Ki67 expression in $>14 \%$ of tumor cells was noted in $36.9 \%$. According to the molecular classification done using the IHC surrogates, the composition of the cohort was as follows: luminal-A 29.9\%, luminal-B(HER2-) $21.4 \%$, luminal-B(HER2+) 14.9\%, HER2-enriched 11.0\%, TNBC $17.5 \%$, and basal-like 5.2\%. TMA cores of 46-49 patients (for each analysis) were lost during microtomy or staining, thereby limiting the number available for molecular classification.

The study subjects have been treated for breast cancer according to the current guidelines. Out of the 99 patients with ER-positive breast cancers, 87 patients had received endocrine therapy. The majority, 90.3\% (149/165) of the study cohort had received chemotherapy. Trastuzumab was initiated for $53 \%$ of subjects who were positive for HER2 (23/43). Mastectomy with level II axillary clearance has been undertaken for $91.3 \%(178 / 195)$ of patients. Postmastectomy radiotherapy has been administered to $69.7 \%$ $(99 / 142)$ of patients.

\section{CAI5-3 assessment}

The pre-surgical blood samples of three patients were hemolyzed and could not be subjected to CA15-3 assessment. Three- and 6-month post-surgical blood samples were obtained from 76 patients. Only 32 and 37 patients turned up for the 9-month and 1-year post-surgical blood sampling, respectively. The mean CA15-3 values of pre- and postsurgical samples are given in Table 1 .

Elevated ( $\geq 30 \mathrm{U} / \mathrm{mL}$ ) pre-surgical CA15-3 was present in $35.9 \%$ of patients. It was $>100 \mathrm{U} / \mathrm{mL}$ in $5.7 \%$ of patients. The prevalence of elevated CA15-3 reduced to $14.3 \%$ at 3 months after mastectomy, increased until the sixth month, and declined thereafter. The mean CA15-3 values of the cohort too declined in a similar pattern over 1 year (Table 1 ).

Post-surgical CA15-3 at 3 months following mastectomy was elevated at least by one unit from the initial value in 35/77 patients, where 11 patients had a $>10 \%$ increase from the pre-surgical value. A reduction in CA15-3 in the 3-month sample was noted in $46 / 77$ patients - most had $<10 \%$ reduction, whereas only $13 / 77$ had $>10 \%$ reduction from the presurgical value; a significant reduction (30\%) was seen only in $5.3 \%$ patients $(4 / 76)$.

Table I CAI5-3 levels with time since mastectomy

\begin{tabular}{|c|c|c|c|c|c|c|}
\hline CAI5-3 & $\begin{array}{l}\text { Number } \\
\text { of patients }\end{array}$ & $\begin{array}{l}\% \text { with elevated } \\
\text { CAI } 5-3\end{array}$ & $\begin{array}{l}\text { Minimum } \\
\text { CAI5-3 level }\end{array}$ & $\begin{array}{l}\text { Maximum } \\
\text { CAI5-3 level }\end{array}$ & Mean & SD \\
\hline Pre-surgical & 192 & 35.9 & 2.34 & 251.00 & 37.227 & 47.043 \\
\hline 3-month & 77 & 14.3 & 4.60 & $2 \mid 0.31$ & 22.805 & 24.944 \\
\hline 6-month & 77 & 31.2 & 5.45 & 251.00 & 28.261 & 29.297 \\
\hline 9-month & 32 & 21.9 & 8.67 & 62.40 & 24.096 & 14.284 \\
\hline 12-month & 37 & 16.2 & 6.26 & 95.60 & 22.800 & 15.685 \\
\hline
\end{tabular}

Note: Cutoff, $30 \mathrm{U} / \mathrm{mL}$. 
Pre-surgical CA15-3 had a significant association only with the size of the tumor ( $p=0.047)$; the percentage of breast cancers with elevated pre-surgical CA15-3 increased with the increasing size of the tumor (T1 20.0\%, T2 $34.8 \%$, and T3 48.5\%). The prevalence of elevated CA15-3 increased with an increase in the TNM stage but did not reach statistical significance ( $p>0.05$; stages I $22.7 \%$, II $31.2 \%$, and III $42.6 \%$ ).

Elevated pre-surgical CA15-3 was seen across all molecular subtypes. Although there was no statistical significance, it was most prevalent among TNBC (48.0\%) and HER2enriched (47.1\%) breast cancer and least prevalent among luminal subtypes (luminal-A 30.4\%, luminal-B(HER2) 24.2\%, luminal-B(HER2+) 30.4\%). ER, PR, and HER2 overexpression did not have a significant association with pre-surgical CA15-3 level.

Elevated 3-month post-surgical values for CA15-3 had a significant association with the molecular subtypes (luminal-A 47.8\%, luminal-B(HER2-) 9.1\%, luminalB(HER2+) 25.0\%, HER2-enriched 50.0\%, TNBC 6.7\%, and basal-like $50.0 \%$ breast cancer subtypes; $p=0.031$ ). The 6-month post-surgical CA15-3 showed an association with the tumor size similar to pre-surgical values $(p=0.037)$. As the 9-month and 1-year post-surgical blood samples were small in number, Fisher's exact test was conducted to check for any associations. The 1-year post-surgical CA15-3 level was elevated when HER 2 was positive $(p<0.001)$ and when Ki67 was expressed in $\geq 14 \%$ of the tumor cells ( $p=0.031$ ). The 9-month CA15-3 value had no association with any of the other parameters assessed (age, grade, tumor size, TNM stage, Ki67, CK5/6, EGFR, hormone receptor, and Claudin expression).

\section{Survival analysis}

Out of the 195 patients, only 182 patients returned for follow-up at the oncology clinic of the teaching hospital from where we enrolled the patients. Twenty-six patients had locoregional recurrence $(2 / 26)$, distant metastasis $(21 / 26)$, or contralateral breast cancer (3/26) during the follow-up period. Patients who did not have elevated pre-surgical CA15-3 ( $\geq 30 \mathrm{U} / \mathrm{mL}$ ) had a better short-term DFS, but it did not reach statistical significance ( $p=0.069$; Figure 1$)$. DFS analysis was repeated with three categories of pre-surgical CA15-3 $(<30,30-100$, and $>100 \mathrm{U} / \mathrm{mL})$ that revealed a significant association ( $p=0.041$; Figure 2 ). Patients with CA15-3 $>100 \mathrm{U} / \mathrm{mL}$ had the worst DFS outcome, whereas those who had normal levels had a better DFS during the short period of follow-up.

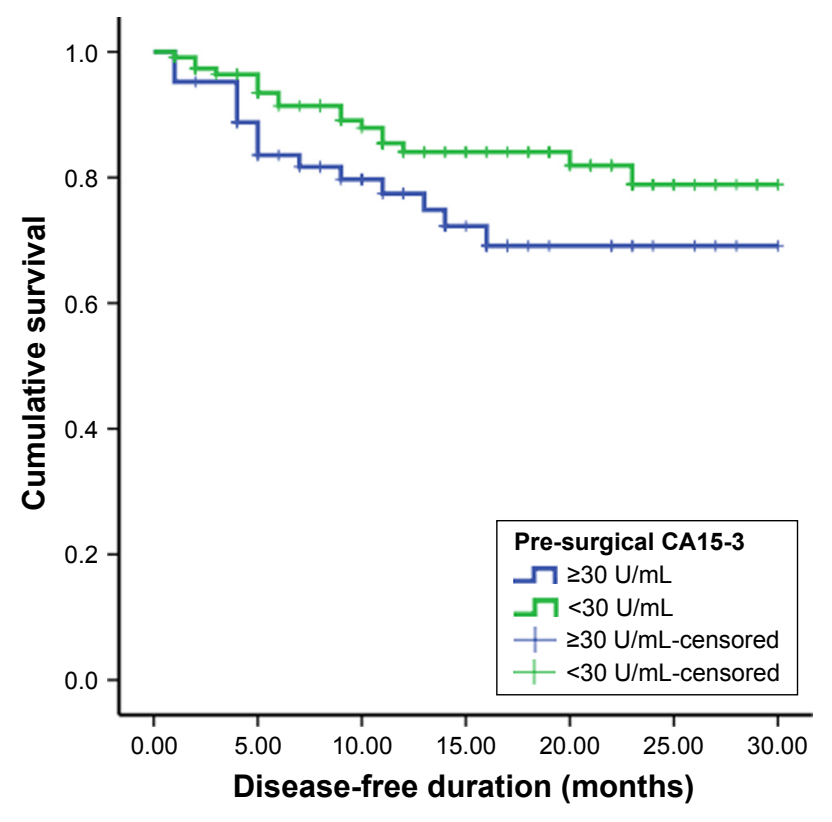

Figure I DFS of patients with breast cancer and elevated pre-surgical CAI5-3 $\geq 30 \mathrm{U} / \mathrm{L}$ against those who had normal levels of CAI5-3.

Univariate analysis was conducted, and factors with a $p<0.1$ were considered for inclusion in the multivariate analysis. ER and PgR expression had a significant association with the DFS on univariate analysis, but, on multivariate analysis, only ER expression was found to have an independent effect on the DFS of the cohort (Table 2).

\section{Discussion}

Assessment of serum tumor markers is a useful tool in the diagnosis, treatment monitoring, and early detection of

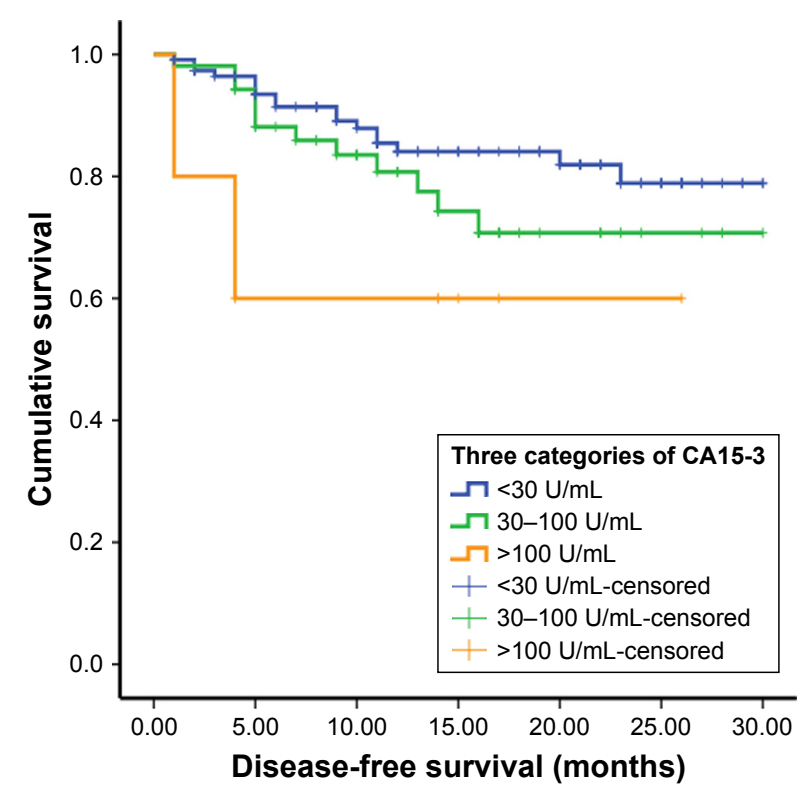

Figure 2 DFS of the three categories according to the pre-surgical CAI5-3 levels. 
Table 2 Univariate and multivariate analyses of factors predicting DFS

\begin{tabular}{lll}
\hline Clinicopathological feature & $\begin{array}{l}\text { Univariate } \\
\text { analysis } \\
\mathbf{p} \text {-value }\end{array}$ & $\begin{array}{l}\text { Multivariate } \\
\text { analysis } \\
\mathbf{p} \text {-value }\end{array}$ \\
\hline ER expression & $\mathbf{0 . 0 2 5}$ & $\mathbf{0 . 0 3 4}$ \\
PgR expression & $\mathbf{0 . 0 2 7}$ & \\
HER2 overexpression & 0.182 & \\
Nottingham grade & 0.103 & \\
Presence/absence of lymph node & 0.052 & \\
metastasis & & \\
Lymph node stage & 0.059 & \\
Tumor size & 0.299 & \\
Age at presentation & 0.310 & \\
Lymphovascular invasion & $\mathbf{0 . 0 1 3}$ & \\
Pre-surgical CAI5-3 $(\geq /<30 \mathrm{U} / \mathrm{mL})$ & 0.069 & \\
\hline
\end{tabular}

Note: Bold values indicate statistical significance $(p<0.05)$.

Abbreviations: DFS, disease-free survival; ER, estrogen receptor; PgR, progesterone receptor.

recurrences of malignant tumors. CA15-3 is the most widely used serum tumor marker for breast cancer. ${ }^{6,10}$ The European Group on Tumor Markers has recommended serial measurement of CA15-3 for detecting recurrence in patients without evidence of disease, and, further, states that it should be done if the detection of recurrent or metastatic disease would alter the clinical management. ${ }^{16}$ However, the American Society of Clinical Oncology does not recommend the use of CA15-3 for monitoring patients for recurrence after primary breast cancer therapy, claiming that the data available in support for a possible recommendation are insufficient. ${ }^{7,9}$ Both guidelines do not recommend the use of CA15-3 as a screening tool. The European Group on Tumor markers recommends the use of preoperative elevated levels of CA15-3 as a prognostic marker in combination with adverse outcome in patients with breast cancer. ${ }^{16}$

CA15-3 is elevated in 70\% of metastatic breast cancer and is much lower (3\%) in localized disease. ${ }^{17}$ A study done in China which included a sample comparable to our study cohort revealed a prevalence of $45.6 \%$. Prevalence as low as $13.6 \%$ has also been reported. ${ }^{18}$

Our study cohort with patients without metastatic disease had a prevalence of $35.9 \%$, which is close to the prevalence reported by Guadagni et al (33\%). ${ }^{19}$ Because only one-third of patients have pre-surgical elevation of CA15-3, its use as a screening tool to detect breast cancers is further disproved by our study.

The highest prevalence of elevated pre-surgical CA15-3 was seen in HER2-enriched and TNBC subtypes in our study. These subtypes are generally known to have a poor prognosis. However, a study done by Yerushalmi et al revealed almost the reverse, where elevated pre-surgical CA15-3 was most common among the luminal subtype..$^{20}$ Their study has been conducted in a sample of patients with metastatic disease, unlike in our study. The 6-month CA15-3 was elevated mostly in the HER2-enriched and luminal subtypes, and this elevation was statistically significant. This observation is difficult to explain with the available data.

Our data reconfirms the association of elevated presurgical CA15-3 with the larger size of the tumor described by other investigators. ${ }^{10,21}$ This association is true for the 6-month post-surgical value as well, according to our findings.

Our data show an elevation in the mean serum CA15-3 in the pre-surgical samples of the cohort that declines to normal 3 months after surgery. The 6-month CA15-3 value is elevated, but a decline is seen thereafter until the 1-year follow-up. Moreover, the 3-month samples had elevated CA15-3 compared to the pre-surgical value in 35/77 patients (45.45\%), although the mean CA15-3 value of the cohort was low. This post-surgical elevation, including the 6-month elevation in CA15-3, may be attributable to chemotherapy-induced apoptosis and necrosis of residual tumor cells because, by the third month, chemotherapy has been initiated. By the sixth month, patients have received a considerable proportion of the recommended chemotherapy cycles. However, sustained elevation of CA15-3 appears to be more frequent with poor prognostic groups of our cohort: those that were HER2 positive and had a high Ki67 index. The post-surgical CA15-3 at 3 months following mastectomy was found to be elevated at least by one unit from the initial value in $35 / 77$ patients in our cohort, and this could be attributed to analytical variations as ELISA was conducted batch wise.

CA15-3 is found to have an independent effect on the survival of patients with breast cancer, in both node-negative and node-positive disease. An independent effect of CA15-3 on breast cancer prognosis has been documented by some studies. ${ }^{17,21}$ Duffy et al reported that CA15-3 had a significant effect on the DFS and, in fact, was found to have an effect independent of the tumor size, axillary node status, and patient age. ${ }^{11}$ The median follow-up period has been 6.27 years in the Duffy et al study, whereas we have addressed only the short-term survival. Similarly, Shao et al reported an independent predictive effect of elevated CA15-3 again in a cohort of patients who had been followed up for a longer period (median 80 months, range 6-118 months). ${ }^{18}$ Lee et al described a similar finding after a longer follow-up (median 72, range 0.8-143.6 months). ${ }^{21}$

Only ER had an independent effect on the DFS in our cohort. Most of the patients in this cohort were ER positive 
and received endocrine therapy. The recurrence rate of ER-positive breast cancers is generally low during the immediate post-surgical follow-up. Therefore, the better survival observed with the ER-positive tumors in our cohort during the short period we followed them up may be attributed to the expected better short-term survival of patients with ER-positive breast cancers.

Pre-surgical CA15-3 was elevated in patients with larger tumors and tumors expressing HER2 and basal marker, and those with higher proliferation activity. Therefore, our data prove that elevated pre-surgical CA15-3 is associated with poor prognostic factors even in the non-metastatic setting. In addition, it is associated with poor short-term DFS, although this effect is dependent on the routinely used prognostic parameters of breast cancer. Our results also show that higher CA15-3 values ( $>100 \mathrm{U} / \mathrm{mL}$ ) have worse DFS outcome.

Elevated CA15-3 levels decline during the post-surgical 1 -year period in most patients, but elevations are seen during the chemotherapy - most likely, due to the destruction of undetected residual micro-metastasis.

\section{Conclusion}

Elevated pre-surgical CA15-3 is a predictor of poor shortterm DFS of patients with breast cancer who present without distant metastasis. However, the predictive capacity is not independent of other prognostic parameters. Therefore, its use is recommended to be undertaken along with other routine prognostic markers.

\section{Acknowledgments}

This work was supported by the Ministry of Science, Technology and Research, Sri Lanka (grant no MTR/TRD/ AGR/3/1/9, Indo-Sri Lanka Joint Research Program 2012).

The authors acknowledge Dr Thusharie Liyanage, Mrs. GGDD Gunawardhane, and the staff of the Department of Pathology, Faculty of Medicine, University of Ruhuna for the support extended; the staff of the Oncology Unit, Teaching Hospital Karapitiya, Galle, Sri Lanka, for permitting the retrieval of follow-up details; and the staff of all general and onco-surgical units of the Teaching Hospital Karapitiya, Sri Lanka, for the support extended in enrolling patients to the study.

\section{Disclosure}

SNG, NL, and DA received a monthly stipend from the funding agency in the form of Research Assistant's allowances. The authors report no other conflicts of interest in this work.

\section{References}

1. Duffy MJ. CA 15-3 and related mucins as circulating markers in breast cancer. Ann Clin Biochem. 1999;36(Pt 5):579-586.

2. Shimizu M, Yamauchi K. Isolation and characterization of mucin-like glycoprotein in human milk fat globule membrane. J Biochem. 1982;91: 515-524.

3. Kufe DW. Mucins in cancer: function, prognosis and therapy. Nat Rev Cancer. 2009;9(12):874-885.

4. Taylor-Papadimitriou J, Burchell J, Miles DW, Dalziel M. MUC1 and cancer. Biochim Biophys Acta. 1999;1455(2-3):301-313.

5. Al-azawi D, Kelly G, Myers E, et al. CA 15-3 is predictive of response and disease recurrence following treatment in locally advanced breast cancer. BMC Cancer. 2006;6:220.

6. Duffy MJ, Evoy D, McDermott EW. CA 15-3: uses and limitation as a biomarker for breast cancer. Clin Chim Acta. 2010;411(23-24): 1869-1874.

7. Harris L, Fritsche H, Mennel R, et al; American Society of Clinical Oncology. American Society of Clinical Oncology 2007 update of recommendations for the use of tumor markers in breast cancer. J Clin Oncol. 2007;25(33):5287-5312.

8. Daniele A, Divella R, Trerotoli P, et al. Clinical usefulness of cancer antigen 15-3 in breast cancer patients before and after surgery. Open Breast Cancer J. 2013;5:1-6.

9. Van Poznak C, Lyndsay N, Harris MD, Somerfield MR. Use of biomarkers to guide decisions on systemic therapy for women with metastatic breast cancer: American Society of Clinical Oncology Clinical Practice Guideline. J Oncol Pract. 2015;11:514-516.

10. Martín A, Corte MD, Alvarez AM, et al. Prognostic value of preoperative serum CA15-3 levels in breast cancer. Anticancer Res. 2006; 26(5B):3965-3971.

11. Duffy MJ, Duggan C, Keane R, et al. High preoperative CA 15-3 concentrations predict adverse outcome in node-negative and node-positive breast cancer: study of 600 patients with histologically confirmed breast cancer. Clin Chem. 2004;50(3):559-563.

12. Goldhirsch A, Winer EP, Coates AS, et al; Panel members. Personalizing the treatment of women with early breast cancer: highlights of the St Gallen International Expert Consensus on the primary therapy of early breast cancer 2013. Ann Oncol. 2013;24(9):2206-2223.

13. Tang P, Tse GM. Immunohistochemical surrogates for molecular classification of breast carcinoma: a 2015 update. Arch Pathol Lab Med. 2016;140(8):806-814.

14. Schnitt SJ. Classification and prognosis of invasive breast cancer: from morphology to molecular taxonomy. Mod Pathol. 2010;23(Suppl 2): S60-S64.

15. Rakha EA. Pitfalls in outcome prediction of breast cancer. J Clin Pathol. 2013;66(6):458-466.

16. Molina R, Barak V, van Dalen A, et al. Tumor markers in breast cancerEuropean group on tumor markers recommendations. Tumor Biol. 2005; 26(6):281-293.

17. Duffy MJ. Biochemical markers in breast cancer: which ones are clinically useful? Clin Biochem. 2001;34(5):347-352.

18. Shao Y, Sun X, He Y, Liu C, Liu H. Elevated levels of serum tumor markers CEA and CA15-3 are prognostic parameters for different molecular subtypes of breast cancer. PLoS One. 2015;10(7):e0133830.

19. Guadagni F, Ferroni P, Carlini S, et al. Re-evaluation of carcinoembryonic antigen (CEA) as a serum marker for breast cancer: a prospective longitudinal study. Clin Cancer Res. 2001;7(8):2357-2362.

20. Yerushalmi R, Tyldesley S, Kennecke H, et al. Tumour markers in metastatic breast cancer subtypes: frequency of elevation and correlation with outcome. Ann Oncol. 2012;23(2):338-345.

21. Lee JS, Park S, Park JM, Cho JH, Kim SI, Park BW. Elevated levels of preoperative CA 15-3 and CEA serum levels have independently poor prognostic significance in breast cancer. Ann Oncol. 2013;24(5): 1225-1231. 
International Journal of Women's Health

Dovepress

\section{Publish your work in this journal}

The International Journal of Women's Health is an international, peerreviewed open-access journal publishing original research, reports, editorials, reviews and commentaries on all aspects of women's healthcare including gynecology, obstetrics, and breast cancer. The manuscript management system is completely online and includes Visit http://www.dovepress.com/testimonials.php to read real quotes from published authors.

Submit your manuscript here: http://www.dovepress.com/international-journal-of-womens-health-journal 